\title{
Control de sistemas ternarios con dos corrientes de recirculado
}

\section{Ternary Systems Control with Two Recycle Steams}

\author{
Solar-González R. \\ Departamento de Ingeniería Química \\ Universidad del Istmo, Oaxaca, México \\ E-mail:solgr@sandunga.unistmo.edu.mx \\ Monroy-Loperena R. \\ ROMON, México \\ E-mail:monroyloperena@hotmail.com \\ Álvarez-Ramírez J. \\ Departamento de Ingeniería de Procesos e Hidráulica \\ Universidad Autónoma Metropolitana-Iztapalapa, Mexico \\ E-mail:jjar@xanum.uam.mx
}

(Recibido: agosto de 2007; aceptado: marzo de 2010)

\section{Resumen}

En la literatura se han planteado estructuras de control en paralelo para el control del diseño de plantas completas. En este trabajo, se considera el comportamiento de una planta que consiste en un sistema de recirculación, un reactor y dos columnas de destilación, cuyo objetivo principal es mejorar la capacidad de recuperación del proceso controlado ante perturbaciones externas. La idea es modificar las condiciones de operación del reactor y la segunda columna, a fin de distribuir el esfuerzo del control de composición ante perturbaciones en la composición de entrada. Para este fin, se propone una estructura de control paralelo donde la composición sea controlada mediante la manipulación simultánea de la velocidad de vapor del rehervidor y la temperatura del reactor. De esta forma, el uso de la temperatura del reactor con una entrada de control secundaria reducirá el comportamiento oscilatorio y la cantidad de flujo de vapor usado.

Descriptores: control planta completa, material recirculado, control paralelo, control de composición.

\footnotetext{
Abstract

In the literature, the use of parallel control structure to improve the control behavior of plantwide designs has been study. In this work, we consider the behavior of a plant that consists of a recycle system, a reactor and two distillation columns where the control aim is to improve the disturbance rejection capabilities of the controlled process. The idea is to change the operating conditions in both the reactor and the second distillation column in order to distribute the composition control effort in the face of fresh feed composition disturbances. To this end, a parallel control structure is proposed where the product composition is regulated by means of simultaneous feedback manipulations of the vapor boilup rate of
} 
DOI: http://dx.doi.org/10.22201/fi.25940732e.2010.11n4.034

Control de sistemas ternarios con dos corrientes de recirculado

the second column and the reactor temperature. In this way, the use of the reactor temperature as a secondary control input reduces oscillatory behavior and the vapour flowrate usage.

Keywords: Plantwide control, material recycle, parallel control, composition control.

\section{Introducción}

Este trabajo amplía los resultados dados por Monroy et al. (2004) para el mejoramiento de procesos recirculados mediante la incorporación de estructuras de control en paralelo (considerado redundante). Específicamente, este trabajo se enfoca en la reacción de los componentes y para formar el producto. La estrategia de control debe contener dos corrientes de alimentación y dos corrientes de recirculado, puesto que existe una conversión de un paso incompleta para ambos reactantes.

Como Tyreus y Luyben (1993) suponen un reactor simple perfectamente mezclado seguido por una sección de separación. Las volatilidades de las especies químicas $A, B$ y $C$ caracterizan la corriente a ser recirculada. Se considera el caso donde la volatilidad del componente $C$ sea intermedia entre los componentes $A$ y $B$. Esto produce un diagrama de flujo del proceso con dos columnas y dos corrientes recirculadas como se esquematiza en la figura 1. La volatilidad de los componentes se supone sea $\alpha_{A}=4, \alpha_{B}=1$ y $\alpha_{C}=2$. El componente $B$, el más pesado, se recircula de los fondos de la primera columna al reactor. El componente $A$, el más ligero, se recircula de la parte superior de la segunda columna al reactor. La figura 1 muestra la nomenclatura usada en este trabajo. La reacción química es $A+B \rightarrow C$ y la velocidad de reacción se supone sea de primer orden en cada reactante:

$$
R_{C}=V_{R} k z_{A} z_{B}
$$

donde:

$R_{C}$ es la velocidad de reacción (lbmol de componente producido por hora),

$V_{R}$ es el volumen en el reactor (lbmol),

$k$ es la velocidad de reacción especifica $\left(h^{-1}\right), z_{A}$ y

$z_{B}$ son las concentraciones de los componentes y en el reactor (en fracción mol), respectivamente. Como fue señalado por Tyreus y Luyben (1993), las "moles" no se conservan en este sistema, debido a que la reacción no es equimolar.

En las estructuras de control propuestas hasta ahora, las variables extensivas (por ejemplo, velocidades de flujo) se usan para balancear el trabajo a partes iguales entre las unidades del proceso, para los cambios en las corrientes de entrada. Esto es similar a la aproximación de Georgakis (1986), donde las variables intensivas se mantienen constantes para diferentes condiciones de operación. La distribución del trabajo de control se realiza mediante la búsqueda conveniente del par uno-a-uno de la salida de control y entrada manipulada. Por otro lado, en todos los sistemas ternarios con esquemas de control con dos corrientes de recirculado reportadas en la literatura, la temperatura del reactor se mantiene constante, manipulando la velocidad de flujo de

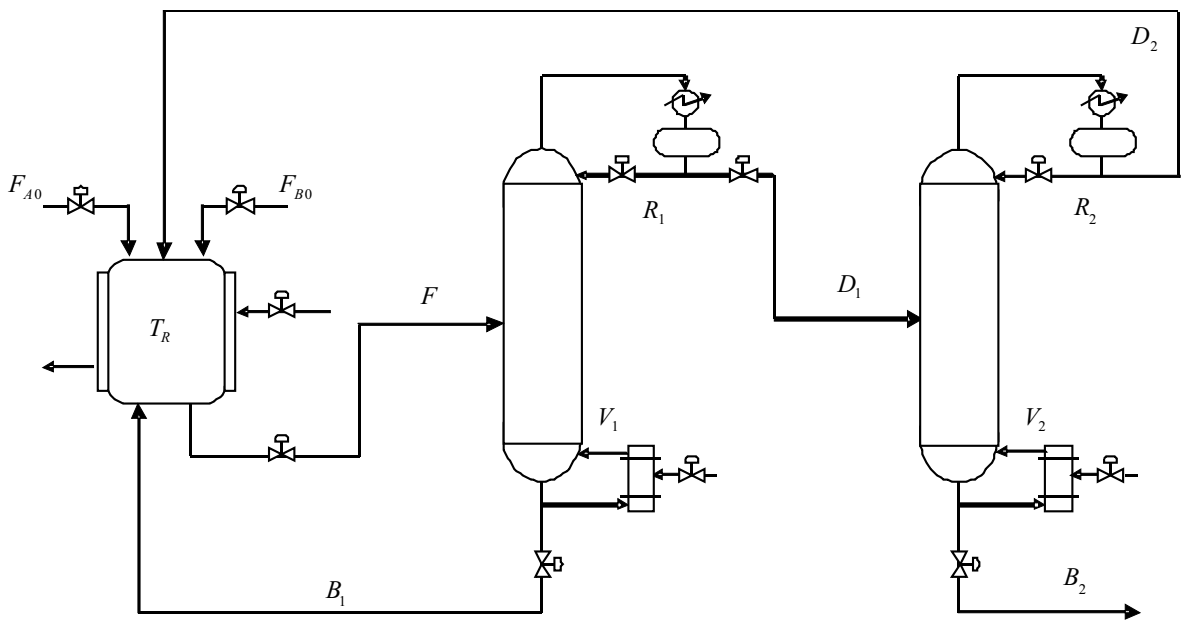

Figura 1. Esquema de un sistema ternario con dos corrientes de recirculado. 
enfriamiento de la chaqueta (Chen et al., 2003). Sin embargo, la temperatura del reactor es una variable intensiva que puede variarse (a través de una manipulación retroalimentada) a fin de inducir cambios de composición significativos con relativamente pequeños esfuerzos de control en estado estable. La temperatura del reactor puede considerarse como un grado de libertad a explotar dentro de un rango de operación seguro para mejorar el funcionamiento del proceso controlado.

Un problema interesante es estudiar cómo la temperatura del reactor disminuirá el esfuerzo de control dedicado por las estructuras de control, basadas solamente en variables extensivas.

Este trabajo se enfoca en este problema explorando una alternativa de estructura de control para controlar sistemas ternarios con dos corrientes de recirculado. Las principales características del esquema de control propuesto son las siguientes:

1. La temperatura del reactor se usa como una entrada manipulada para el propósito de control retroalimentado. De esta forma, el esquema de control usa tanto variables manipuladas extensivas como intensivas.

2. Una estructura de control paralelo (también llamado habituado), se usa para distribuir el esfuerzo de control entre el reactor y la segunda columna de destilación. La idea es manipular simultáneamente la temperatura del reactor y la velocidad de vapor en la segunda columna para regular la composición del producto del proceso. Como resultado, las perturbaciones en la composición de las corrientes de entrada se reducen por medio de relativamente pequeños cambios en la temperatura del reactor.

En este artículo mediante el uso de simulaciones rigurosas se muestra el comportamiento del controlador de composición frente a perturbaciones en la corriente de alimentación.

\section{Control planta completa convencional}

Uno de los problemas más importantes en este tipo de control es la selección de una estructura de control (es decir, un par entrada/salida) que garantice una estabilización satisfactoria y la regulación efectiva de propiedades. Como se mencionó anteriormente, la estructura de control se basa en esquemas de pares uno-a-uno, esto es, una entrada manipulada se usa para regular una salida. A fin de proponer una estructura de control redundante (ó rectangular) para mejorar el funcionamiento, en este trabajo partimos de la estructura de control no redundante propuesta por Cheng et al. (2003), donde el volumen del reactor $\left(V_{R}\right)$ se supone constante. La estructura de control tiene las siguientes características:

1) La velocidad de producción está dado por la alimentación de $A\left(F_{0 A}\right)$.

2) El volumen del reactor se mantiene constante controlando la velocidad de flujo del efluente $(F)$.

3) La relación de recirculado de la primera columna se fija $\left(B_{1} / F_{o A}\right)$.

4) La composición de fondos de la primera columna $\left(V_{1} / B_{1}\right)$ se controla modificando la relación del rehervidor en la primera columna de destilación.

5) El nivel de fondos de la primera columna se controla manipulando la alimentación del reactante $B\left(F_{o B}\right)$.

6) La relación de reflujo de la primera columna se fija $\left(R_{1} / D_{1}\right)$.

7) La composición del producto $\left(x_{B_{2}, C}\right)$ se mantiene manipulando la relación del rehervidor de la segunda columna $\left(V_{2} / B_{2}\right)$.

8) La relación de reflujo de la segunda $\left(R_{2} / D_{2}\right)$ se fija.

Nótese que sólo las variables extensivas se usan como entradas manipuladas. En particular, la temperatura del reactor se mantiene constante. El razonamiento tras de la estructura de control de Cheng et al. (2003), es obtener un esfuerzo de control balanceado distribuido en el diagrama de flujo del proceso mientras que se satisfacen los requerimientos de calidad del producto $\left(x_{B_{2}, C}\right)$.

La figura 2 presenta la respuesta del proceso controlado bajo la estructura de control de Cheng et al. (2003). La planta fue diseñada como sugieren Elliot et al. (1996), donde se supone tenemos densidad y peso molecular constantes, flujo equimolar, platos teóricos, condensador total y rehervidor parcial.

La acumulación y la constante de tiempo hidráulica se calcularon de la formula de Francis, suponiendo 1-in de altura de presa. Todas las corrientes de alimentación fueron líquido saturado.

El tanque de reflujo y las bases de las columnas son de un tamaño que proporcione 5 min de acumulación como resultado de las respectivas velocidades de flujo en estado estacionario. Los valores obtenidos por el diseño se usan como valores nominales y se reportan en la tabla 1. Las mediciones de retardo para el flujo, la temperatura y composición fueron tomadas como $0.1,1$ y 6 min, respectivamente. 

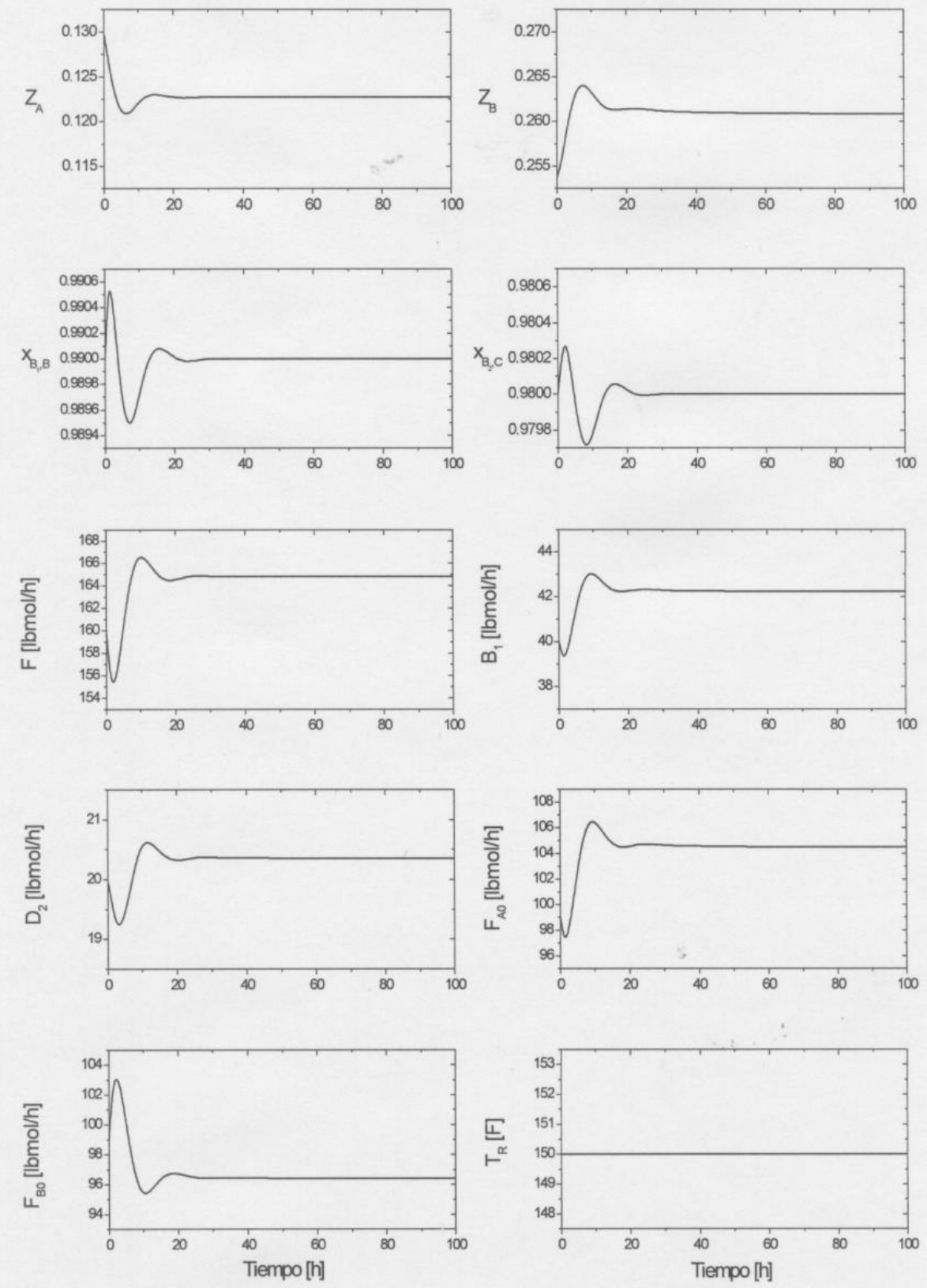

Figura 2. Comportamiento del proceso controlado bajo un esquema de control convencional (es decir, cuadrático). El proceso está bajo los efectos de una perturbación en la composición de alimentación, $z_{0 A, A}: 1.0 \rightarrow 0.92$ y $z_{0 A, B}: 1.0 \rightarrow 0.08$ 
Tabla 1. Valores nominales para la configuración del caso estudiado

\begin{tabular}{|c|c|c|}
\hline$V_{R}$ & Volumen del reactor $[\mathrm{lbmol}]$ & 2973.69 \\
\hline$k_{0}$ & Velocidad de reacción $\left[h^{-1}\right]$ & 247526.94 \\
\hline$E_{A}$ & Energía de activación $[\mathrm{Btu} / \mathrm{lbmol}]$ & 15000 \\
\hline$T_{R}$ & Temperatura del reactor $[F]$ & 150 \\
\hline$F_{A 0}$ & Flujo de alimentación del componente $A[\mathrm{lbmol} / \mathrm{h}]$ & 99 \\
\hline$F_{B 0}$ & Flujo de alimentación del componente $B[\mathrm{lbmol} / \mathrm{h}]$ & 99 \\
\hline$F$ & Flujo de salida del reactor $[\mathrm{lbmol} / \mathrm{h}]$ & 160 \\
\hline$z_{A}$ & Fracción mol del componente $A$ en la alimentación & 0.1299 \\
\hline$z_{B}$ & Fracción mol del componente $B$ en la alimentación & 0.2538 \\
\hline$N_{1}$ & $\begin{array}{l}\text { Número de etapas ideales incluyendo condensador y rehervidor en la } \\
\text { columna } 1\end{array}$ & 28 \\
\hline$N F_{1}$ & Etapa de alimentación en la columna 1 & 12 \\
\hline$D_{1}$ & Flujo de destilado de la columna $1[\mathrm{lbmol} / \mathrm{h}]$ & 120 \\
\hline$R_{1}$ & Reflujo de la columna $1[\mathrm{lbmol} / \mathrm{h}]$ & 177.03 \\
\hline$V_{1}$ & Vapor de ebullición de la columna $1[\mathrm{lbmol} / \mathrm{h}]$ & 297.03 \\
\hline$B_{1}$ & Flujo de fon dos de la columna $1[\mathrm{lbmol} / \mathrm{h}]$ & 40 \\
\hline$x_{B 1, A}$ & $\begin{array}{l}\text { Fracción mol del componente } A \text { en el producto de fondos de la } \\
\text { columna } 1\end{array}$ & 0.00 \\
\hline$x_{B 1, B}$ & $\begin{array}{l}\text { Fracción mol del componente } B \text { en el producto de fondos de la } \\
\text { columna } 1\end{array}$ & 0.99 \\
\hline$N_{2}$ & $\begin{array}{l}\text { Número de etapas ideales incluyendo condensador y rehervidor en la } \\
\text { columna } 2\end{array}$ & 28 \\
\hline$N F_{2}$ & Etapa de alimentación en la columna 2 & 18 \\
\hline$D_{2}$ & Flujo de destilado de la columna $2[\mathrm{lbmol} / \mathrm{h}]$ & 20 \\
\hline$R_{2}$ & Reflujo de la columna $2[\mathrm{lbmol} / \mathrm{h}]$ & 145.87 \\
\hline$V_{2}$ & Vapor de ebullición de la columna $2[\mathrm{lbmol} / \mathrm{h}]$ & 165.87 \\
\hline$B_{2}$ & Flujo de fondos de la columna $2[\mathrm{lbmol} / \mathrm{h}]$ & 100 \\
\hline$x_{B 2, A}$ & $\begin{array}{l}\text { Fracción mol del componente } A \text { en el producto de fondos de la } \\
\text { columna } 2\end{array}$ & 0.01 \\
\hline$x_{B 2, A}$ & $\begin{array}{l}\text { Fracción mol del componente } B \text { en el producto de fondos de la } \\
\text { columna } 2\end{array}$ & 0.01 \\
\hline
\end{tabular}


Todos los lazos de control consisten de un compensador PI basado en modelos de respuesta escalón (primer orden más tiempo de retardo), y sintonizados con guías tipo IMC, reportadas por Skogestad (2003) con constantes de tiempo a lazo cerrado tomadas como máx. $\left\{0.75 \tau_{0}, \theta\right\}$, donde $\tau_{0}$ es la constante de tiempo a lazo abierto y $\theta$ es el retardo del lazo. Nótese que, además de que se obtiene la regulación de la composición del producto, la respuesta presenta un comportamiento oscilatorio, el cual puede deberse al efecto en la regulación de $x_{B_{2}, C}$ basado sólo en manipulaciones de la velocidad de vapor de la segunda columna de destilación. De esta forma, el lazo de control es incapaz de proveer una respuesta rápida para las perturbaciones en la alimentación, lo cual afecta al proceso con una subsiguiente falta de balance del trabajo en los diferentes equipos. En la siguiente sección, mostraremos que puede obtenerse un mejoramiento en el control incorporando la temperatura del reactor, como una variable secundaria manipulada para regular la composición del producto en colaboración con la relación del rehervidor de la segunda columna, $\left(V_{2} / B_{2}\right)$.

\section{Control planta completa mediante aproximación en paralelo}

La excesiva carga en el equipo de vaporización para compensar la presencia de perturbaciones en el flujo de alimentación a la planta, es inducida por un desequilibrio del esquema de control convencional. Esto es, ante la presencia de la perturbación en el flujo de alimentación el esquema convencional ajusta las condiciones de operación sólo en la columna de destilación final incrementando el flujo de vapor. Dada la estructura de control convencional, tal ajuste se requiere para compensar los incrementos en la composición del flujo de alimentación a la planta, lo cual es inducido por decrementos en la conversión del reactor que son debidos principalmente a una reducción en el tiempo de residencia (inducidos a su vez por incrementos en el flujo de alimentación). Variando la temperatura del reactor tendremos un efecto en estado estacionario del proceso sobre la conversión.

De esta forma, si una cantidad excesiva de trabajo es detectada en la columna de destilación, manifestándose en velocidades de vaporización significativas, debe hacerse un ajuste en la temperatura del reactor, lo cual, a su vez, producirá un cambio en la composición de alimentación de la columna. Esta acción de control deberá aliviar el esfuerzo de operación dedicado por el proceso de separación. Para este fin, se hacen las siguientes consideraciones: a) La temperatura del reactor $T_{R}$ se manipula para regular la calidad del producto $x_{B_{2}, C}$.

b) Una vez que el comportamiento correctivo de $T_{R}$ ha sido especificado a través de una función de retroalimentación $T_{R}=\phi_{M}\left(x_{B_{2} C}\right)$, la temperatura del reactor se modifica por medio de una arquitectura de control cascada en serie donde la función $\phi_{M}\left(x_{B_{2}, C}\right)$ juega el rol de controlador maestro y las acciones del controlador estándar PI como el controlador esclavo (Luyben, 1990).

Para diseñar un controlador retroalimentado que manipule simultáneamente la temperatura del reactor $T_{R}$ y la velocidad de vapor $V_{2}$, se formula un modelo entrada/salida no cuadrático de $2 \times 1$ como sigue:

$$
y(s)=G_{1}(s) u_{1}(s)+G_{2}(s) u_{2}(s)
$$

en donde por conveniencia, se usa la siguiente notación: $y(s)=\Delta x_{B_{2}, C}(s)$ es la salida regulada, $u_{1}(s)=\Delta V_{2}(s)$ y $u_{2}(s)=\Delta T_{R}(s)$ son las entradas manipuladas.

De esta forma, el problema es diseñar un controlador retroalimentado en paralelo

$$
u(s)=C(s) e_{y}(s)
$$

donde $u(s)=\left[u_{1}(s), u_{2}(s)=\left[C_{1}(s), C_{2}(s)\right]\right.$ y $e_{y}(s)=y_{s p}(s)$ $-y(s)$ que es la regulación del error, tales que $e_{y} \rightarrow 0$ asintóticamente. Nótese que el resultado de la manipulación del controlador retroalimentado en paralelo forma entradas de control $u_{1}(s)$ y $u_{2}$ para regular la salida simple $y(s)$. De las respuestas al escalón alrededor del punto de operación nominal, encontramos que la dinámica entrada/salida puede ser modelada como un proceso de primer orden descrito por:

$$
\begin{aligned}
& G_{1}(s)=\frac{K_{V_{2} x_{B_{2}} C}}{\tau_{V_{2}} s+1} \exp \left(-\theta x_{B_{2}, C} s\right) \\
& y \\
& G_{2}(s)=\frac{K_{T_{R} x_{B_{2}} C}}{\tau_{T_{R}} s+1} \exp \left(-\theta x_{B_{2}, C} s\right)
\end{aligned}
$$

Esto es, la dinámica entrada/salida $V_{2} \rightarrow x_{B_{2}, C}$ y $T_{R} \rightarrow x_{B_{2}, C}$ se modela como un proceso de primer orden estable. El operador de tiempo de retardo $\left(-\theta x_{B_{2} C} s\right)$ contabiliza los retardos por las mediciones de composición y efectos de transporte.

La idea esencial tras el control en paralelo es explotar las características específicas y objetivos de operación de 
un proceso con dos tipos diferentes de variables manipuladas: (a) una lenta, de tipo económico y (b) una rápida de tipo costoso (Henson, 1995). En nuestro caso, el flujo de vapor en la segunda columna, $V_{2}$, juega el rol de la variable manipulada rápida y costosa, mientras que la temperatura del reactor, $T_{R}$ será la variable lenta y económica.

Esto es, dado que la operación del reactor involucra procesos de calentamiento/enfriamiento y los equipos de separación requieren vapor de ebullición, es de esperarse que la manipulación del flujo de vapor sea más costosa que la manipulación de la temperatura del reactor. Esto debido a que la relación $C_{P} / \lambda_{V}$ (capacidad calorífica/calor de vaporización) es de cerca de 0.01 en casos típicos. En general, en la industria química es más costoso vaporizar que calentar (dentro de cierto rango de temperatura práctico) un líquido.

Así, debe esperarse que cambios pequeños en la temperatura de reacción durante la operación tengan un impacto significativo en la carga de vaporización requerida para los procesos de separación. De esta forma, el esfuerzo de control aplicado a la regulación de la composición del producto del proceso será distribuido entre la manipulación de la temperatura del reactor que es económica, pero de respuesta lenta, y la manipulación de la velocidad de flujo de vapor que es costosa, pero de respuesta rápida.

El problema de diseñar controladores retroalimentados para sistemas rectangulares ha sido tratado anteriormente (Henson, 1995 y Monroy et al., 2004). Algunos enfoques parten de la regulación de la presión sanguínea en los mamíferos (Henson, 1995). Otro reporte del autor está basado en ideas de factorización (Monroy et al., 2004). En este trabajo, proponemos una aproximación heurística basada en una segmentación convexa de la salida regulada. Esto se hace describiendo la salida regulada como:

$$
y(s)=y_{1}(s)+y_{2}(s)
$$

donde $y_{1}(s)=\beta y(s), y_{2}(s)=(1-\beta) y(s)$ son salidas virtuales, y $\beta \in[0,1]$ es la segmentación o parámetro habituado. De (1), podemos escribir

$$
\begin{aligned}
& y_{1}(s)=\beta G_{1}(s) u_{1}(s) \\
& y_{2}(s)=(1-\beta) G_{2}(s) u_{2}(s)
\end{aligned}
$$

De esta forma, si el objetivo de control es $y(s) \rightarrow y_{s p}(s)$, donde $y_{s p}(s)$ es la señal de punto de referencia, el objetivo de control del sistema de control segmentado (4) es

$$
y_{1}(s) \rightarrow y_{s p, 1}(s) \text { y } y_{2}(s) \rightarrow y_{s p, 2}(s)
$$

donde los puntos de referencia correspondientes son $y_{1}(s)=\beta y(s)$ y $y_{2}(s)=(1-\beta) y(s)$. Puesto que ambos objetivos de control dados en la ecuación (5) se alcanzan, entonces $y(s) \rightarrow y_{s p}(s)$ está garantizado. Nótese que, puesto que la ecuación (4) es un sistema de control desacoplado y las funciones de transferencia $G_{1}(s)$ y $G_{2}(s)$ son estables, el objetivo de control se alcanza con dos compensadores PI descentralizados (sintonizados como en una configuración de control no redundante).

Nótese que cuando $\beta=1$ solo $u_{1}(s)$ está presente, esto es, la velocidad de vapor $V_{2}$ es el conjunto de valores nominales $\bar{V}_{2}$ y toda la dinámica y esfuerzo de control estacionario son brindados por el control de temperatura del reactor. Contrariamente, cuando $\beta=0$ solo $u_{2}(s)$ estará presente, esto es, la temperatura del reactor se mantiene en su valor nominal $\bar{T}_{R}$ y toda la dinámica y esfuerzo de control estacionario son ejecutados por el controlador de la segunda columna. Esto corresponde al esquema de control convencional descrito en la sección anterior. Cuando $0<\beta<1$, ambos controladores tienen una contribución no trivial para la regulación del flujo de composición en los fondos $x_{B_{2}, C}$. De esta forma, el esquema de control paralelo propuesto tiene la estructura de un controlador balanceado para $0<\beta<1$. Conforme $\beta$ se incrementa, el reactor ejecutará más trabajo de procesamiento, llevando menor esfuerzo de control (es decir, menor uso de vapor) a la segunda columna de destilación.

\section{Simulaciones numéricas}

Como en el caso cuadrático, se obtuvieron valores adecuados para el diseño del controlador paralelo de la respuesta al escalón de entrada/salida alrededor del punto de operación nominal y suponiendo un modelo estable de primer orden, que se reportan en la tabla 2 . El comportamiento de la configuración de control convencional ante una perturbación en la composición de alimentación del $8 \%$, en donde la composición de la corriente de alimentación $F_{0 A}$, va de $z_{0 A, A}: 1.0 \rightarrow 0.92$ y $z_{0 A, C}: 0.0 \rightarrow 0.08$ es mostrado en la figura 2 . En estas simulaciones, la temperatura del reactor $T_{R}$ se controla a su valor nominal de $150^{\circ} \mathrm{F}$, tal que el sistema de control sólo ajusta las condiciones de operación de la segunda columna para mantener las especificaciones del producto $x_{B_{2}, C}$ en la corriente de fondos a su valor deseado. Nótese que al modificarse la composición de los componentes en el flujo de alimentación de $F_{0 A}$ ocasionarán cambios en la temperatura del reactor y en el volumen, 
Tabla 2. Parámetros de los controladores

\begin{tabular}{cccc}
\hline $\begin{array}{c}\text { Variable } \\
\text { controlada }\end{array}$ & $\begin{array}{c}\text { Variable } \\
\text { manipulada }\end{array}$ & $\begin{array}{c}\text { Ganancia en estado } \\
\text { estacionario }\end{array}$ & $\begin{array}{c}\text { Constante de tiempo } \\
\text { dominante }\end{array}$ \\
\hline$V_{R}$ & $F$ & -2.75 & 30.81 \\
$x_{B 1, B}$ & $V_{1}$ & $4.65 \times 10^{-4}$ & 3.84 \\
$x_{B 2, B}$ & $V_{2}$ & $2.17 \times 10^{-4}$ & 6.30 \\
$x_{B 1, C}$ & $T_{R}$ & $5.99 \times 10^{-4}$ & 5.30 \\
\hline
\end{tabular}
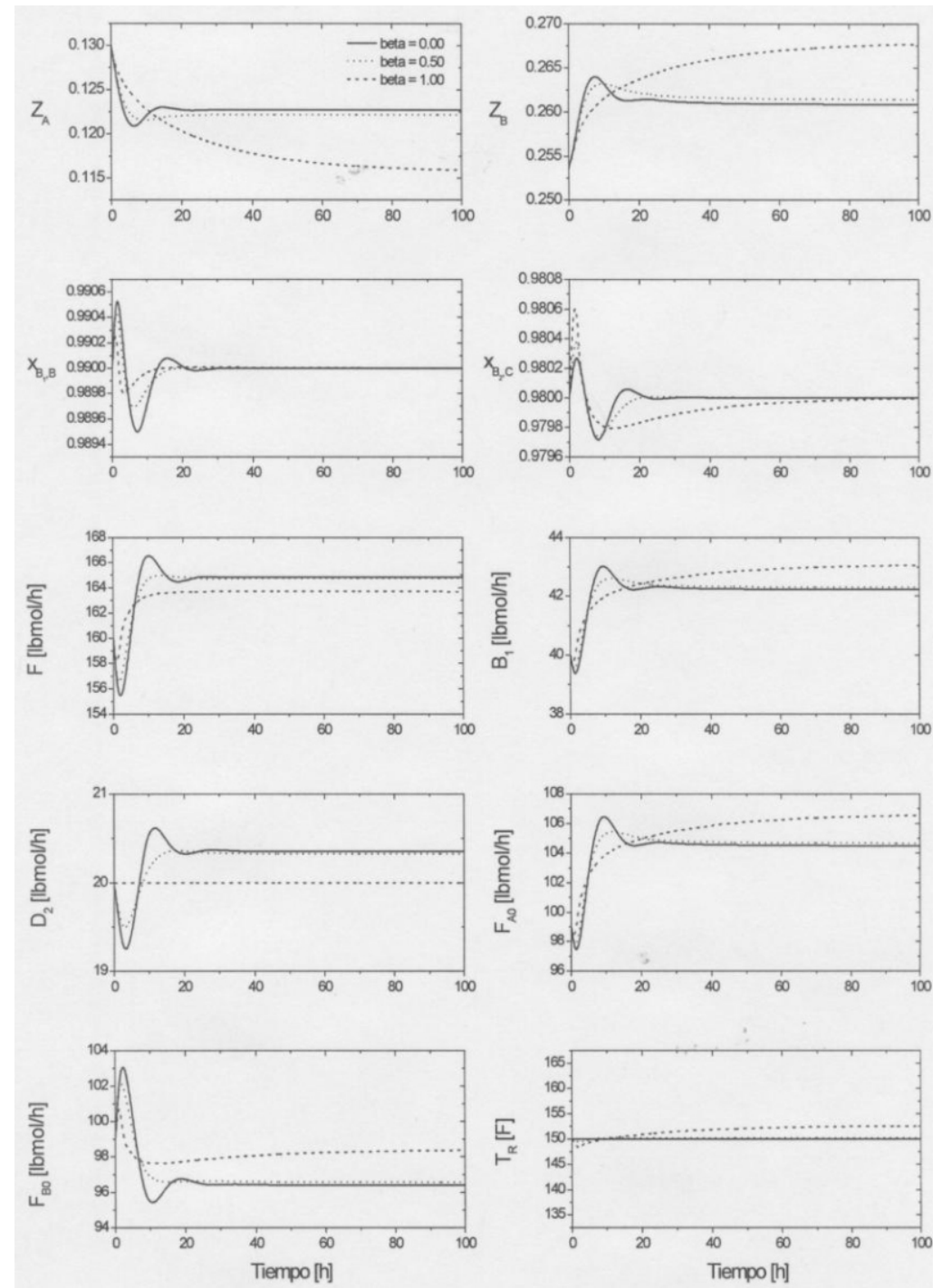

Figura 3. Comportamiento del proceso controlado bajo un esquema de control en paralelo (es decir, rectangular). El proceso está bajo los efectos de una perturbación en la composición de alimentación, $z_{0 A, A}: 1.0 \rightarrow 0.92$ y $z_{0 A, B}: 1.0 \rightarrow 0.08$. Nótese que el proceso usa menor velocidad de vapor en la segunda columna con mayor trabajo de procesamiento (es decir, $\beta$ se incrementa) realizado por el reactor. 
lo que en consecuencia modificará la composición. Para mantener la composición especificada del producto en la columna 2, la carga de vapor deberá incrementarse, lo cual induce a un posterior incremento en la corrientes de recirculación $B_{1}$ y $D_{2}$.

En la figura 3 se presenta nuevamente el comportamiento de la configuración de control convencional (cuando $\beta=0$ ) a fin de comparar con la configuración de control propuesta. Primeramente, se presenta el caso cuando la composición del producto del componente $C$ se controla sólo por medio de la temperatura del reactor (es decir, $\beta=1$ ) observándose que el tiempo de convergencia en la composición del componente $C$ en los fondos de la segunda columna es mucho mayor que el mostrado por el control convencional. Note que los flujos $F, B_{1}, D_{2}, F_{0 A}$ y $F_{0 B}$ tienen un comportamiento dinámico amortiguado. Sin embargo, la temperatura del reactor se modifica ligeramente de $150^{\circ} \mathrm{F}$ a $148.5^{\circ} \mathrm{F}$, en las primeras $5 h$, para posteriormente incrementarse lentamente a un valor de $152.5^{\circ} \mathrm{F}$ en $80 \mathrm{~h}$. La composición del componente $B$ en el producto de fondos de la primera columna presenta una menor oscilación en comparación de la configuración convencional, además alcanza el punto de referencia en menor tiempo, cerca de $20 \mathrm{~h}$. Contrariamente, la composición del componente $C$ en el producto de fondos de la segunda columna presenta un sobredisparo más pronunciado en las primeras 5 horas; sin embargo, no presenta posteriores oscilaciones convergiendo en un tiempo aproximado de $100 \mathrm{~h}$.

Esto muestra que el uso de la temperatura del reactor como variable manipulada para controlar la composición del componente $C$ en el producto de fondos de la columna 2 proporciona un comportamiento lento, pero estable de toda la planta. Como se observa en la figura 3 , el uso del control paralelo propuesto con $\beta=0.5 \mathrm{com}$ bina las características deseadas del esquema de control convencional y el esquema de control de temperatura del reactor, proporcionando un comportamiento de control más eficaz que el esquema de control convencional. Basándose en un solo parámetro simple $(\beta)$ es capaz de proporcionar un esfuerzo de control distribuido entre la operación del reactor (a través de transformaciones químicas) y la operación de la segunda columna (a través de separaciones físicas). A mayor valor de $\beta$, mayor el trabajo de procesamiento ejecutado por el reactor.

De esta forma, una vez que el lazo de control simple se sintoniza, $\beta$ será el único parámetro que deberá sintonizarse para obtener una distribución conveniente del procesamiento de trabajo. Una selección sistemática del parámetro sintonizado $\beta$ debe estar basada en la inclusión de un criterio adicional, por ejemplo, consideraciones económicas y de seguridad.

\section{Conclusiones}

Resumiendo, presentamos un esquema de control paralelo para una planta con sistemas ternarios y dos corrientes de recirculado. La idea básica fue manipular simultáneamente la velocidad de flujo de vapor en la segunda columna de destilación y la temperatura del reactor, esperando que el procesamiento de trabajo sea distribuido en ambos equipos.

El esquema de control propuesto se diseñó por medio de metodologías de control paralelo para distribuir en forma sistemática, la cantidad de esfuerzo de control de las diferentes unidades del proceso. Las simulaciones numéricas demostraron la habilidad del esquema de control resultante para manipular las perturbaciones en la condiciones de alimentación.

En sentido global, confirmamos que la conclusión obtenida para una planta reactor/separador/recirculado (Monroy et al., 2004), en la que el problema de selección de la estructura de control y diseño correspondiente a los compensadores retroalimentados para el control de planta completa involucra una configuración de control novedosa y la estructura de los compensadores (por ejemplo, control paralelo, compensadores de baja frecuencia, etc.) explotan la estructura específica del proceso. Hecho esto, se espera que la operación de planta completa sea más eficiente y se obtenga un funcionamiento de control más robusto.

\section{Referencias}

Cheng Y.C., Yu C.C. Optimal Region for Design and Control of Ternary Systems. AIChE J., 49:682, 2003.

Elliot T.R., Luyben W.L. Quantitative Assessment of Controllability During the Design of a Ternary System with Two Recycle Streams. Ind. Eng. Chem. Res., 35:3470, 1996.

Georgakis C. On the Use of Extensive Variables in Process Dynamics and Control. Chem. Eng. Sci., 41:1471, 1986.

Henson M.A., Ogunnaike B.A., Schwaber J.S. Habituating Control, Strategies for Process Control. AIChE J. 13:291, 1995.

Luyben W.L. Process Modeling Simulation and Control for Chemical Engineers. New York. McGraw-Hill. 1990.

Monroy-Loperena R., Solar R., Alvarez-Ramirez J. Balanced Control Scheme for Reactor/Separador Processes with Material Recycle. Ind. Eng. Chem. Res., 43:1853, 2004. 
DOI: http://dx.doi.org/10.22201/fi.25940732e.2010.11n4.034

Control de sistemas ternarios con dos corrientes de recirculado

Skogestad S. Simple Analytical Rules for Model Reduction and PID Controller Tuning. J. Process Control., 13:291, 2003.
Tyreus B.D., Luyben W.L. Dynamics and Control of Recycle Systems. 4. Ternary Systems with One and Two Recycle Streams. Ind. Eng. Chem. Res., 32:1154, 1993.

Semblanza de los autores

Rocío Solar-González. En 2003, obtuvo su doctorado en ciencias (ingeniería química) en la Universidad Autónoma Metropolitana unidad Iztapalapa en el Distrito Federal, México. Actualmente es profesor-investigador en el Instituto de Energía de la Universidad del Istmo en Oaxaca y miembro del SNI. Su investigación se relaciona con la dinámica y control de procesos químicos.

Rosendo Monroy-Loperena. Profesor investigador egresado de la Universidad Autónoma Metropolitana en el Distrito Federal, México. Sus principales intereses en investigación son el desarrollo de modelos y estrategias de control en el área de procesos.

José Álvarez-Ramírez. Es miembro de la Asociación Mexicana de Control Automático (AMCA). Actualmente se desarrolla como profesor investigador en la Universidad Autónoma Metropolitana en el Distrito Federal, México. Es miembro del Sistema Nacional de Investigadores con Nivel III. Sus líneas de investigación se encuentran enfocadas a las áreas de matemáticas aplicadas y teoría de control. 